Revista Brasil. Bot., V.32, n.1, p.95-108, jan.-mar. 2009

\title{
Biologia reprodutiva de Byrsonima gardnerana A. Juss. (Malpighiaceae) e interações com abelhas Centris (Centridini) no Nordeste do Brasil ${ }^{1}$
}

\author{
ELISANGELA SANTANA BEZERRA ${ }^{2,4}$, ARIADNA VALENTINA LOPES ${ }^{3} \mathrm{e}$ \\ ISABEL CRISTINA MACHADO ${ }^{3}$
}

(recebido: 17 de janeiro de 2008; aceito: 04 de dezembro de 2008)

\begin{abstract}
Reproductive biology of Byrsonima gardnerana A. Juss. (Malpighiaceae) and interactions with Centris bees (Centridini) in northeastern Brazil). The pollination ecology, the reproductive system and the flowering phenology of Byrsonima gardnerana A. Juss were studied in a Caatinga area of the Parque Nacional do Catimbau, Pernambuco state, Brazil. The species is a shrub with hermaphrodite and zygomorphic flowers, which change color after pollination, with oil as primary floral resource. Flowering pattern is annual, lasting 4-5 months, occurring along the dry season. The species is self-incompatible, has elevated pollen viability and high pollen/ovule ratio. Eleven species of bees were observed visiting the flowers of B. gardnerana, nine of them behaving as pollinators (seven Centris spp.) and two as pollen thieves. Bees of the genus Centris were the most frequent and effective floral visitors, collecting oil and also pollen, in this case vibrating the anthers, always contacting the reproductive structures of the flowers. The flowers of B. gardnerana are, therefore, an important resource to specialized native bees that feed their larvae with floral oils, hence guarantying frequent visits to the flowers.
\end{abstract}

Key-words - Caatinga, oil-flowers, phenology, Restinga, self-incompatibility

RESUMO - (Biologia reprodutiva de Byrsonima gardnerana A. Juss. (Malpighiaceae) e interações com abelhas Centris (Centridini) no Nordeste do Brasil). A polinização, o sistema reprodutivo e a fenologia da floração de Byrsonima gardnerana A. Juss foram estudados em uma área de Caatinga situada no Parque Nacional do Catimbau, Agreste de Pernambuco. Byrsonima gardnerana é uma espécie arbustiva com flores hermafroditas e zigomorfas, que mudam de cor em decorrência da polinização. O recurso floral primário é o óleo. O padrão de floração é anual, durando 4-5 meses, ao longo da estação seca. A espécie é auto-incompatível, apresenta alta viabilidade polínica e elevada razão pólen/óvulo. Foram observadas 11 espécies de abelhas visitando as flores de B. gardnerana, nove delas atuando como polinizadoras (sete espécies de Centris) e duas como pilhadoras de pólen. As espécies de Centris foram as mais freqüentes e efetivas, coletando óleo e pólen, neste caso vibrando as anteras, sempre contatando as estruturas reprodutivas das flores. Flores de B. gardnerana constituem, portanto, importante fonte de alimento para as abelhas especializadas nativas, cuja necessidade dos óleos florais na composição da dieta de suas larvas garante constantes visitas.

Palavras-chave - auto-incompatibilidade, Caatinga, fenologia, flores de óleo, Restinga

\section{Introdução}

Flores que oferecem óleo como recompensa para seus polinizadores ocorrem em nove famílias de Angiospermas, sendo duas de Monocotiledôneas (Iridaceae e Orchidaceae) e sete de Eudicoteledôneas sensu APG II (Calceolariaceae, Cucurbitaceae, Krameriaceae, Malpighiaceae, Plantaginaceae, Primulaceae e Solanaceae), somando 79 gêneros e,

1. Parte da dissertação de mestrado da primeira autora, Programa de Pós-Graduação em Biologia Vegetal, Universidade Federal de Pernambuco.

2. Universidade Federal de Pernambuco, Programa de Pós-Graduação em Biologia Vegetal, Rua Prof. Nelson Chaves s/n, 50372-970 Recife, PE, Brasil.

3. Universidade Federal de Pernambuco, Departamento de Botânica, Rua Prof. Nelson Chaves s/n, 50372-970 Recife, PE, Brasil.

4. Autor para correspondência: eli.lucia@ig.com.br aproximadamente, 2.400 espécies distribuídas por todo o mundo (Buchmann 1987, Machado 2002, 2004, Alves dos Santos et al. 2007).

Sendo óleo um atrativo primário (Anderson 1979, Vogel 1990), secretado por glândulas especializadas denominadas elaióforos (Vogel 1974, Simpson \& Neff 1983), as flores de óleo têm como principais polinizadores abelhas fêmeas da tribo Centridini, que utilizam este recurso na dieta de suas larvas, sendo consideradas seus polinizadores-chave (e.g. Buchmann 1987, Vogel 1990, Vogel \& Machado 1991, Michener 2000, Machado 2002, 2004, Machado et al. 2002, Alves dos Santos et al. 2007).

As abelhas da tribo Centridini são especializadas na coleta de óleos florais que é mediada por um conjunto de pêlos especiais localizados geralmente no basitarso das pernas anteriores e medianas (e.g. Vogel 1974, Neff \& Simpson 1981, Simpson \& Neff 1981, Gottsberger 
1986, Buchmann 1987, Michener 2000, Machado 2002, 2004). A similaridade no comportamento de coleta do recurso por estas abelhas é bastante conhecido, sendo citado por vários autores como forte indício de co-evolução com as flores de óleo de Malpighiaceae (Anderson 1979, Simpson \& Neff 1981, Buchmann 1987, Vogel 1990, Machado 2002, 2004).

A família Malpighiaceae possui $c a .60$ gêneros e 1.100 espécies predominantemente tropicais, dos quais aproximadamente 47 gêneros e 950 espécies encontramse exclusivamente no continente americano, apresentando hábitos que variam de herbáceos perenes a arbustivos e arbóreo de grande porte, sendo, entretanto, as lianas o grupo mais expressivo (Anderson 1979, Sazima \& Sazima 1989, Vogel 1990). As espécies apresentam estrutura floral, horário de antese e coloração das pétalas relativamente homogêneos, todos indicativos da síndrome da melitofilia (Anderson 1979, Faegri \& Pijl 1979, Gottsberger 1986, Vogel 1990).

Com relação ao gênero Byrsonima, estudos acerca da ecologia da polinização são encontrados para vários ecossistemas: Cerrado (Gottsberger 1986, Barros 1992, Gaglianone 2000), Floresta Atlântica (Teixeira \& Machado 2000), Floresta Estacional Semidecidual (Sazima \& Sazima 1989, Vinson et al. 1997, Sigrist \& Sazima, 2004), Restinga (Albuquerque \& Rêgo 1989, Rêgo \& Albuquerque 1989, Freitas et al. 1999, Silva \& Martins, 1999; Ramalho \& Silva 2002) e Savana Amazônica (Benezar \& Pessoni 2006). Porém, citações para estudos realizados em Caatinga são inexistentes, sendo encontradas apenas referências em trabalhos de levantamento de espécies de abelhas Centris (Aguiar \& Martins 1997, Zanella 2000a, b) e sobre a flora explorada por estas abelhas (Martins 1994, 1995, Aguiar et al. 1995, 2003).

Este trabalho teve como objetivo analisar a fenologia da floração, a morfologia e biologia floral e o sistema reprodutivo de Byrsonima gardnerana A. Juss., bem como suas relações com as abelhas Centridini em uma área de Caatinga em Pernambuco. Foram realizadas ainda observações paralelas em populações da espécie ocorrentes na região litorânea do mesmo Estado, a fim de se comparar a comunidade de polinizadores nos dois ecossistemas, bem como discutir a interação de Apis mellifera com as abelhas autóctones polinizadoras da espécie.

\section{Material e métodos}

Locais de estudo - Os estudos de campo foram realizados no Parque Nacional do Catimbau (Parna Catimbau; 8 ${ }^{\circ} 32^{\prime} 14^{\prime \prime}$ a $8^{\circ} 35^{\prime} 12^{\prime \prime} \mathrm{S}$ e $37^{\circ} 14^{\prime} 42^{\prime \prime}$ a $37^{\circ} 15^{\prime} 02^{\prime \prime} \mathrm{W}$ ), município de Buíque, situado no Agreste Pernambucano, distando $285 \mathrm{~km}$ da capital Recife. As observações foram feitas no período de março/2002 a março/2003, através de visitas mensais com duração de quatro a seis dias, perfazendo um total de 446 horas.

O Parna Catimbau é uma das poucas unidades de conservação de proteção integral em área de Caatinga, abrangendo um polígono de $600,7 \mathrm{~km}^{2}$ ao longo dos municípios de Buíque, Tupanatinga e Ibimirim, PE. Apresenta clima quente e seco (BS'hW - Köppen), solo arenoso/ pedregoso e vegetação variando entre Caatinga típica da Região Semi-Árida e zonas de mosaicos com elementos de Campo Rupestre e Cerrado. A precipitação e temperatura médias anuais giram em torno de $600 \mathrm{~mm}$ e $26{ }^{\circ} \mathrm{C}$, respectivamente, sendo os meses de março a junho o período de chuvas mais intensas na região (Sudene 1990, Rodal et al. 1998) (figura 1).

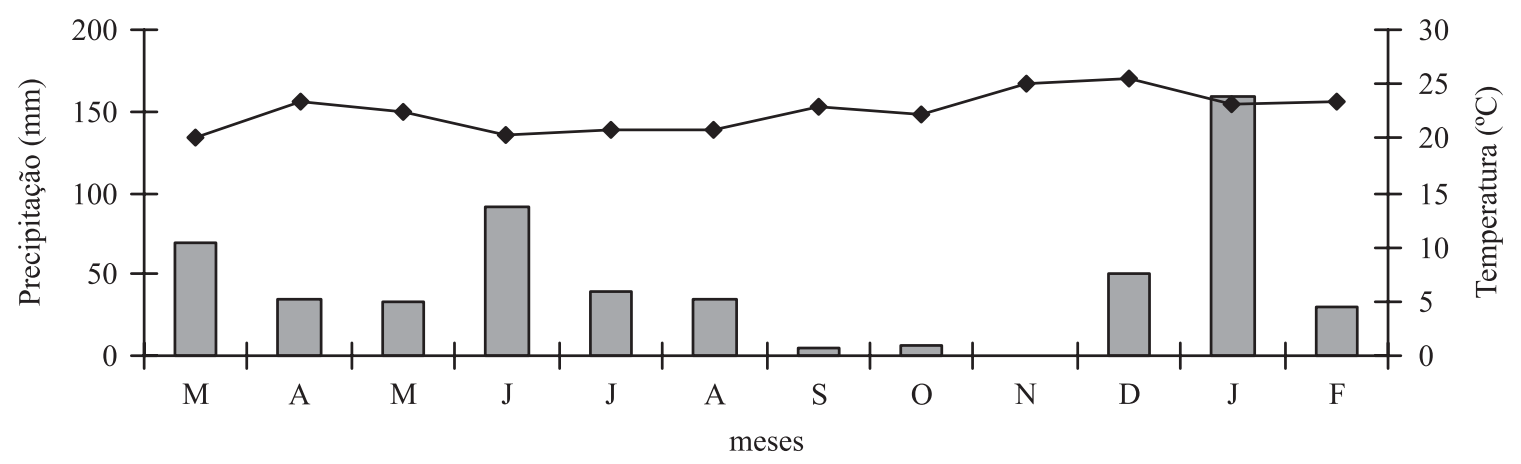

Figura 1. Dados climatológicos da área de estudo durante o período de março/2002 a fevereiro/2003 (fonte: INMET). As barras e linha expressam respectivos valores médios de precipitação e temperatura ocorridos no período.

Figure 1. Climatic data of the study site during the period of March/2002 to February/2003 (source: INMET). The bars and line express respective mean values of haste and temperature occurred in the period. 
Para amostrar possíveis diferenças entre a guilda de abelhas coletoras de óleo relacionadas com a composição vegetacional, três sub-áreas foram escolhidas para a realização das observações de campo: 1. Chapada São José (8³4'61' S; $37^{\circ} 13$ ' 63' W) - pertence à Formação Tacaratu entre as cotas de 900-1.000 m; apresenta vegetação arbustiva perenifólia, marcada por elementos típicos de Campo Rupestre, crescendo em solo de arenito quartzítico e conglomerados (Rodal et al. 1998). 2. Sítio Serra Branca (8³3’23” S; 37¹5’08” W) - distando ca. 7 km da Chapada São José, apresenta vegetação de Caatinga arbustiva e elementos de Cerrado (SNE 2002) e altitude de $885 \mathrm{~m}$. e 3. Cânion ( $8^{\circ} 32^{\prime} 05^{\prime}$ ' S; 37¹5'12” W) - situado a ca. $5 \mathrm{~km}$ da segunda área, apresentando altitude aproximada de $930 \mathrm{~m}$ e vegetação com fisionomia similar a do Sítio Serra Branca.

Observações paralelas em setembro de 2002 foram também realizadas em uma área de Restinga com aproximadamente um hectare, situada próxima ao manguezal do litoral da Praia de Catuama ( $\left.7^{\circ} 33^{\prime} \mathrm{S} ; 3^{\circ} 00^{\prime} \mathrm{W}\right)$, município de Goiana, PE, totalizando 30 horas de observações.

Fenologia da floração - A fenologia da floração foi observada através do acompanhamento mensal de 10 indivíduos marcados em cada uma das três áreas do Parna Catimbau, seguindo a metodologia sugerida por Fournier (1974). O padrão de floração foi determinado de acordo com as classificações propostas por Gentry (1974) e Newstrom et al. (1994).

Biologia floral - Foram feitas observações acerca da morfologia, tamanho, coloração e horário de antese floral, além do número de botões e de flores abertas/inflorescência/ dia. A fim de se verificar a existência de padrões de mudanças florais na espécie e se a polinização acarreta alterações na duração e seqüência da antese (Gori 1983), foram realizados três tipos de experimentos, cada um com cinco flores distribuídas em diferentes indivíduos: 1. flores foram marcadas na fase de botão, permanecendo expostas aos visitantes até 48 horas após o início da antese (tratamento controle). 2. botões, em pré-antese, foram encobertos com sacos de papel semi-permeável e, após sua abertura, as flores foram polinizadas manualmente com pólen de flores de outros indivíduos (polinização cruzada), sendo então reensacadas até a senescência floral. 3. botões, em pré-antese, foram encobertos com sacos de papel semi-permeável, permanecendo intactos até a senescência floral. Todas as flores foram observadas em intervalos de três horas a partir do início de cada tratamento, sendo estas realizadas até às 17 h00 de cada dia.

A receptividade estigmática foi detectada através da reação com permanganato de potássio $0,25 \%$ (Robinsohn 1924). A viabilidade polínica foi estimada através da coloração do citoplasma com carmim acético 2\% (Radford et al. 1974), utilizando 10 flores coletadas aleatoriamente em diferentes indivíduos.

A razão pólen/óvulo (sensu Cruden 1977) foi estimada através da contagem dos grãos de pólen de 15 botões em pré-antese com auxílio de câmara de Neubauer (Moura et al. 1987), preparados de acordo com a metodologia descrita por Lloyd (1972), e da contagem dos óvulos, destes mesmos botões, sob microscópio estereoscópico.

Sistema reprodutivo - O sistema reprodutivo da espécie foi estudado através de experimentos de autopolinização espontânea, autopolinização manual e polinização cruzada, além da verificação de ocorrência ou não de agamospermia (Radford et al. 1974) realizados em oito indivíduos. Em todos os tratamentos os botões foram encobertos em pré-antese com sacos de papel semi-permeável, sendo utilizados um mínimo de 30 botões de diferentes indivíduos para cada teste.

A fim de se observar formação de frutos em condições naturais, 611 flores, distribuídas nas três áreas do Parna Catimbau (cinco indivíduos/área), foram marcadas e acompanhadas até a formação ou não de frutos. Testes de freqüência floral (teste $G$ ) foram aplicados para verificar diferenças na formação natural de frutos entre as diversas áreas de estudo, utilizando-se software BioEstat 3.0 (Ayres et al. 2003).

Visitantes florais - O comportamento dos visitantes florais, a duração, o horário das visitas e o recurso floral por eles procurado foram registrados através de observações visuais diretas no campo, em horários variados ao longo do dia, complementadas através de registros fotográficos em plantas focais. A frequiência de visitas foi analisada durante nove dias, das 5 h00 às 16h00, no pico de floração da espécie, totalizando 99 horas de observação, distribuídas entre a Chapada São José, o Sítio Serra Branca e o Cânion, no Parna Catimbau, e a Restinga de Catuama, na proporção de 3:1:3:2 dias, respectivamente, de acordo com o período de floração de cada sub-área.

Na Chapada São José e no Cânion as observações foram realizadas em cinco indivíduos, com um número médio de 40 flores abertas/indivíduo durante o período. No Sítio Serra Branca, a alta intensidade de visitas de Centris impossibilitou sua quantificação nos indivíduos como um todo, ficando as observações restritas a seis inflorescências.

As abelhas visitantes foram classificadas quanto ao tamanho como (a) pequenas (comprimento $<12 \mathrm{~mm}$ ) ou (b) médio-grandes ( $\geq 12 \mathrm{~mm}$ ) sensu Frankie et al. (1983) e quanto à eficiência na polinização como (a) polinizadores, quando sempre contatavam as estruturas reprodutivas ao coletar o recurso, ou (b) pilhadores, quando não contatavam as estruturas reprodutivas e/ou danificavam as anteras e/ou estigmas, por vezes destruindo-os completamente.

Material biológico - Representantes de cada espécie de visitante floral foram capturados, montados, conservados à seco e enviados a especialistas para identificação, encontrandose depositados na coleção de polinizadores do Laboratório de Biologia Floral e Reprodutiva da UFPE como espécimestestemunho. Material testemunho de Byrsonima gardnerana foi depositado no Herbário da Universidade Federal de Pernambuco como espécime-testemunho (UFP 35.637). 


\section{Resultados}

Fenologia da floração - Foram observadas variações quanto ao início e ao pico de floração de B. gardnerana nas diferentes áreas de estudo (figura 2). No Sítio Serra Branca, os primeiros botões começaram a abrir em setembro, atingindo o pico de produção de flores no mês de dezembro, com um número médio de 18,78 $\pm 4,11$ flores por inflorescência/dia $(n=30)$. O período de floração estendeu-se até o início de fevereiro, quando foram observadas flores esparsas em alguns indivíduos (figura 2).

No Cânion, área com maior concentração de indivíduos, os quais eram os mais altos e frondosos, a média de flores/inflorescência foi de $16,70 \pm 3,40$ $(n=30)$. Nesta área a floração teve início em novembro, sendo encontradas algumas flores esporádicas, atingindo o pico no mês de fevereiro.

Na Chapada São José, as primeiras flores também foram vistas em setembro em apenas dois indivíduos, porém o pico foi atingido mais tardiamente, em dezembro, tendo número médio de flores por inflorescência semelhante $(15,19 \pm 2,98 ; n=30)$.

Hábito e morfologia floral - Byrsonima gardnerana apresenta porte arbustivo em todas as áreas amostradas. As flores são zigomorfas, hermafroditas e dispostas em racemos terminais. A corola possui diâmetro médio de $1,74 \pm 0,07 \mathrm{~cm}$, sendo composta por cinco pétalas livres e alvas, sendo a pétala superior, denominada estandarte, diferenciada por apresentar unguículo alargado. Cada flor contém cinco pares de glândulas epiteliais elípticas produtoras de óleo, todas funcionais, localizadas na face externa de cada sépala. $\mathrm{O}$ androceu é formado por 10 estames cujas anteras são amarelas e apresentam deiscência longitudinal e grãos de pólen brancos e pulverulentos. O gineceu é composto por ovário súpero, unicarpelar e uniovular, apresentando três estiletes livres com estigmas puntiformes.

Sequiência da antese - Em cada inflorescência abrem-se $c a$. duas a três flores por dia, a maioria no início da manhã, entre 5 h00 e 5 h 30 , embora tenham sido observados botões iniciando o processo de abertura em horários variados do dia até o início da tarde.

Os experimentos para verificação da seqüência de antese revelaram diferentes respostas das flores a cada tratamento. No primeiro dia do experimento controle, as flores, com seus estigmas já receptivos e anteras deiscentes, apresentam pétalas e elaióforos alvos. Ao final do segundo dia (ca. 36 horas após o início da antese), as pétalas estão murchas e recurvadas para baixo, com a base avermelhada e as anteras esbranquiçadas e ressecadas. Os elaióforos e a pétala estandarte apresentam, neste momento, coloração avermelhada mais intensa. No início do terceiro dia, pétalas e estames encontram-se ressecados e caem ao toque.

As flores previamente ensacadas, polinizadas manualmente (polinização cruzada), apresentaram duração, coloração e seqüência de antese semelhantes ao tratamento controle (polinização natural), sendo, porém, observada maior resistência da flor em relação às pétalas e estames, permanecendo vistosas (com pétalas estendidas) por $c a$. 60 horas. As flores ensacadas que

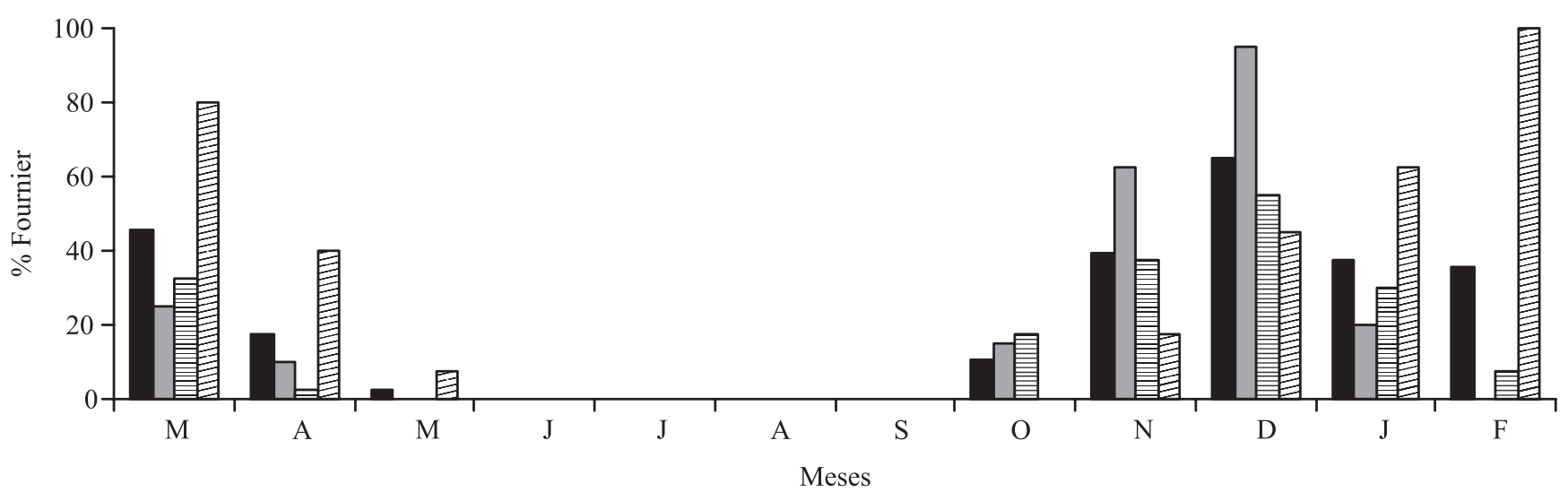

Figura 2. Fenologia de floração de Byrsonima gardnerana no Parque Nacional do Catimbau, região semi-árida de Pernambuco. ( $=$ Geral; $\square=$ Chapada São José; $\boxminus=$ Sítio Serra Branca; $\square=$ Cânion).

Figure 2. Flowering phenology of Byrsonima gardnerana at the Parque Nacional do Catimbau, semi-arid region of Pernambuco state, northeastern Brazil. ( $\square=$ Geral; $\square=$ Chapada São José; 目 = Sítio Serra Branca; $\square=$ Cânion). 
não foram polinizadas nem natural, nem manualmente, duraram aproximadamente 72 horas e sua coloração permaneceu inalterada ao longo de todo período de antese.

Sistema reprodutivo - A viabilidade polínica média foi de 97,8\%; o número médio de grãos de pólen por flor (e conseqüentemente a razão pólen-óvulo, visto que a flor possui um único óvulo) foi de $268.000 \pm 13.832$ grãos para cada óvulo/flor.

Os testes de polinização manual revelaram que Byrsonima gardnerana é auto-incompatível (tabela 1), não havendo formação de frutos nos tratamentos de agamospermia, nem nos de autopolinizações espontânea ou manual.

As flores expostas aos polinizadores revelaram diferenças quanto ao percentual de frutos formados (condições naturais) nas três sub-áreas do Parna Catimbau, sendo a maior taxa de formação natural de frutos encontrada na Chapada São José e em uma das populações do Cânion, seguida pelo Sítio Serra Branca

Tabela 1. Resultados dos experimentos controlados sobre o sistema reprodutivo de Byrsonima gardnerana e das observações de polinização natural nas três áreas de estudo do Parque Nacional do Catimbau, PE. (* = Flores sem visitas de Apis mellifera; ** = Flores com baixa freqüência de $A$. mellifera (< 10 visitas/intervalo de $2 \mathrm{~h} / \mathrm{dia})$; *** = Flores submetidas à intensa atividade de $A$. mellifera (> 50 visitas/ intervalo de $2 \mathrm{~h} / \mathrm{dia})$ ).

Table 1. Results of the treatments to investigate the reproductive system of Byrsonima gardnerana and observations of natural pollination in the three study sites at the Parque Nacional do Catimbau, PE, northeastern Brazil. (* = Flowers without Apis mellifera visits; $* *=$ Flowers with low frequency of $A$. mellifera visitation (< 10 visits/ intervals of $2 \mathrm{~h} /$ day); $* * *=$ Flowers subject to high frequency of A. mellifera visitation (> 50 visits/intervals of $2 \mathrm{~h} /$ day)).

\begin{tabular}{lcc}
\hline Tratamento & Flores $(n)$ & Frutos $(\%)$ \\
\hline Agamospermia & 30 & 0 \\
Autopolinização espontânea & 30 & 0 \\
Autopolinização manual & 30 & 0 \\
Polinização cruzada & 30 & 93,3 \\
Polinização natural & & \\
$\quad$ Chapada São José & $129 *$ & $52,7^{\mathrm{a}}$ \\
Sítio Serra Branca & $50 *$ & $30,0^{\mathrm{b}}$ \\
Cânion & $237^{* *}$ & $23,2^{\mathrm{c}}$ \\
& $195^{* * *}$ & $48,2^{\mathrm{a}}$ \\
\hline
\end{tabular}

Valores na mesma coluna seguidos por letras diferentes foram significativamente diferentes $(P<0,001)$. e pela segunda população analisada no Cânion (tabela 1). Os testes estatísticos mostraram que essas diferenças foram significativas $(G=19,9 ; g l=3 ; P<0,001)$ (tabela 1$)$.

Visitantes florais - Abelhas fêmeas do gênero Centris foram os visitantes florais mais representativos observados em Byrsonima gardnerana tanto nas três sub-áreas de Caatinga, quanto na área de Restinga estudadas (tabela 2). Apesar das diferenças na composição vegetacional das sub-áreas de Caatinga, a guilda das espécies de Centris visitantes de $B$. gardnerana foi semelhante, variando apenas a frequiência de visitas (figura 3). $\mathrm{Na}$ área de Restinga, além das cinco espécies encontradas na Caatinga, foram ainda registradas duas outras (C. nitens e C. leprieuri). Estas abelhas visitavam as flores para coleta de óleo e também pólen, por meio da vibração dos estames.

Outras abelhas procuravam as flores exclusivamente em busca de pólen (tabela 2): Augochloropsis sp., vista nas flores de B. gardnerana apenas na área de Restinga (Praia de Catuama), coletando pólen de forma ativa (vibração); A. mellifera, encontrada apenas no Cânion; Plebeia minima, observada no Sítio Serra Branca coletando pólen residual deixado nas pétalas pelas abelhas grandes ou diretamente nas anteras de $B$. gardnerana, sem entretanto, efetuar a polinização (tabela 2); e Trigona spinipes, presente tanto na Chapada São José quanto no Sítio Serra Branca, coletando pólen. Sua atividade causava sérios danos às estruturas florais reprodutivas, destruindo completamente os estames e, por vezes, os estiletes.

A Chapada São José apresentou o menor número de visitas em relação às demais áreas de estudo. As abelhas iniciavam suas atividades de coleta por volta das $6 \mathrm{~h} 00$, estendendo-se até as 14h00 (figura 3A). No Cânion, na população formada por indivíduos mais jovens, onde a freqüência de visitas pôde ser realizada, esta análise revelou o período de maior atividade ocorrendo entre as 7h00 e 11 h00 (figura 3B), diminuindo gradativamente até as $16 \mathrm{~h} 00$. Nesses indivíduos, $C$. caxiensis foi a abelha mais freqüente, seguida de $C$. aenea, $C$. tarsata, $C$. fuscata, C. flavifrons e Apis mellifera. A freqüência vespertina dos visitantes foi mais acentuada nas flores que se abriam tardiamente, sendo estas preferidas em detrimento das que iniciaram a antese pela manhã.

Por sua vez, nos indivíduos do Cânion que apresentavam maior porte e com mais ramificações, as visitas às flores iniciaram mais cedo, por volta das $5 \mathrm{~h} 00$, sendo observada dezenas de indivíduos de A. mellifera ao mesmo tempo, em uma única planta de $B$. gardnerana. Suas visitas eram relativamente demoradas, levando em 
Tabela 2. Visitantes florais de Byrsonima gardnerana, classificados de acordo com Michener 2000. Local de ocorrência $\left(\mathrm{PNC}=\right.$ Parque Nacional do Catimbau, ${ }^{1} \mathrm{C}$ ânion, ${ }^{2}$ Sítio Serra Branca, ${ }^{3}$ Chapada São José; CAT = Catuama); recurso procurado $(\mathrm{P}=$ pólen, $\mathrm{O}=$ óleo $)$; modo de coleta $(\mathrm{CO}=$ coletora, $\mathrm{MO}=$ mordedora, $\mathrm{RA}=$ raspagem, $\mathrm{VI}=$ vibração sensu Michener 1962, Wille 1963, Buchmann et al. 1977); resultado das visitas ( $\mathrm{PO}=$ polinização, $\mathrm{PI}=$ pilhagem).

Table 2. Floral visitors of Byrsonima gardnerana, classified in accordance with Michener 2000. Site of occurrence $(\mathrm{PNC}=\mathrm{Parque}$ Nacional do Catimbau, ${ }^{1}$ Cânion, ${ }^{2}$ Sítio Serra Branca, ${ }^{3}$ Chapada São José; CAT $=$ Catuama $)$; collected resource $(\mathrm{P}=$ pollen, $\mathrm{O}=$ oil); mode of collection ( $\mathrm{CO}=$ gleanning, $\mathrm{MO}=$ biting, $\mathrm{RA}=$ scraping, $\mathrm{VI}=$ buzzing sensu Michener 1962, Wille 1963, Buchmann et al. 1977); result of visitation $(\mathrm{PO}=$ pollination, $\mathrm{PI}=$ resource theft $)$.

\begin{tabular}{|c|c|c|c|}
\hline Abelhas visitantes & $\begin{array}{l}\text { Local de } \\
\text { ocorrência }\end{array}$ & $\begin{array}{c}\text { Recurso/ } \\
\text { Modo de coleta }\end{array}$ & $\begin{array}{l}\text { Resultado da } \\
\text { visita }\end{array}$ \\
\hline \multicolumn{4}{|l|}{ APIDAE } \\
\hline \multicolumn{4}{|l|}{ Apini } \\
\hline Apis mellifera* Linnaeus, 1758 & $\mathrm{PNC}^{1}$ & $\mathrm{P} / \mathrm{CO}$ & $\mathrm{PO}$ \\
\hline \multicolumn{4}{|l|}{ Centridini } \\
\hline Centris (Centris) aenea** Lepeletier, 1841 & $\mathrm{PNC}^{123 / \mathrm{CAT}}$ & $\mathrm{O} / \mathrm{RA}-\mathrm{P} / \mathrm{VI}$ & $\mathrm{PO}$ \\
\hline C. (C.) caxiensis** Ducke, 1907 & $\mathrm{PNC}^{123} / \mathrm{CAT}$ & $\mathrm{O} / \mathrm{RA}-\mathrm{P} / \mathrm{VI}$ & PO \\
\hline C. (C.) flavifrons ${ }^{* *}$ Fabricius, 1775 & $\mathrm{PNC}^{123 / \mathrm{CAT}}$ & $\mathrm{O} / \mathrm{RA}-\mathrm{P} / \mathrm{VI}$ & $\mathrm{PO}$ \\
\hline C. (C.) leprieuri** Spinola, 1841 & CAT & $\mathrm{O} / \mathrm{RA}-\mathrm{P} / \mathrm{VI}$ & $\mathrm{PO}$ \\
\hline C. (C.) cf. nitens ${ }^{* *}$ Lepeletier, 1841 & CAT & $\mathrm{O} / \mathrm{RA}-\mathrm{P} / \mathrm{VI}$ & $\mathrm{PO}$ \\
\hline C. (Hemisiella) tarsata* (Smith, 1874) & $\mathrm{PNC}^{123} / \mathrm{CAT}$ & $\mathrm{O} / \mathrm{RA}-\mathrm{P} / \mathrm{VI}$ & $\mathrm{PO}$ \\
\hline C. (Trachina) fuscata** Lepeletier, 1841 & $\mathrm{PNC}^{123 / \mathrm{CAT}}$ & $\mathrm{O} / \mathrm{RA}-\mathrm{P} / \mathrm{VI}$ & $\mathrm{PO}$ \\
\hline \multicolumn{4}{|l|}{ Meliponini } \\
\hline Plebeia minima* (Gribodo, 1893) & $\mathrm{PNC}^{2}$ & $\mathrm{P} / \mathrm{CO}$ & PI \\
\hline Trigona spinipes* (Fabricius, 1793) & $\mathrm{PNC}^{23}$ & $\mathrm{P} / \mathrm{MO}$ & PI \\
\hline \multicolumn{4}{|l|}{ HALICTIDAE } \\
\hline \multicolumn{4}{|l|}{ Halictini } \\
\hline Augochloropsis sp.* & CAT & $\mathrm{P} / \mathrm{VI}$ & $\mathrm{PO}$ \\
\hline
\end{tabular}

* abelhas pequenas; ** abelhas médio-grandes sensu Frankie et al. 1983.

média de três até $c a$. cinco minutos. A coleta de pólen era feita com as pernas anteriores e medianas, transferindo-o para as corbículas das pernas posteriores na própria flor. Por um período aproximado de 4 horas (das 5 h00 às 9h00), esta espécie predominava sobre as demais, apresentando comportamento "territorialista" e agonístico, reunindo-se em duas ou três contra as abelhas nativas. Após esse horário, sua presença diminuía sensivelmente ocorrendo visitas rápidas e esporádicas, passando a ser observado o predomínio das Centridini até as 15 h00, quando cessavam as visitas.

Nas populações localizadas no Sítio Serra Branca, C. aenea foi novamente o visitante mais frequiente, assim como na Chapada São José, seguida por $C$. fuscata, $C$. caxiensis, C. tarsata e C. flavifrons. Plebeia minima e Trigona spinipes foram registradas visitando as flores de B. gardnerana nesta área, comportando-se como pilhadoras (tabela 2).
Centris tarsata foi a menor espécie de Centridini observada nas flores de B. gardnerana. O seu tamanho limitava a coleta de óleo apenas aos três pares de glândulas mais próximos da pétala estandarte. Em todas as áreas de estudo, seu período de maior atividade coincidia com o horário em que as abelhas grandes tornavam-se menos frequientes, provavelmente devido à diminuição do volume de óleo secretado.

$\mathrm{Na}$ Restinga, as visitas iniciaram por volta das $5 \mathrm{~h} 00$, destacando-se $C$. aenea, C. flavifrons e $C$. fuscata em número de indivíduos e, por conseguinte, na intensidade de visitas.

As espécies de Centris apresentaram comportamento similar entre si, aproximando-se da flor frontalmente e prendendo-se, através das mandíbulas, ao ungüículo da pétala estandarte. A raspagem dos elaióforos era realizada com as pernas anteriores (que alcançavam as quatro glândulas mais próximas da pétala estandarte) e 

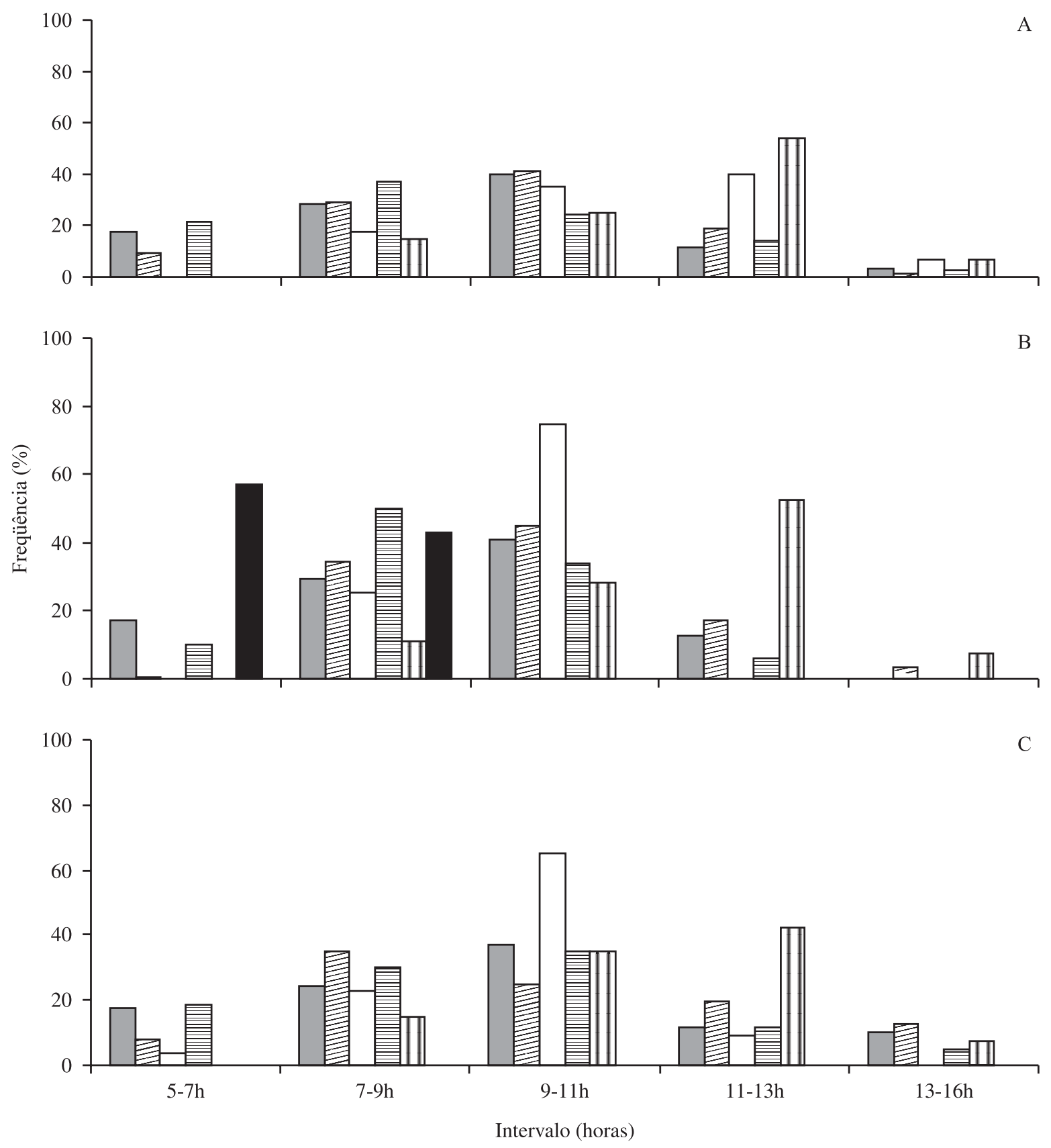

Figura 3. Freqüência de visitas dos polinizadores de Byrsonima gardnerana nas diferentes áreas de estudo do Parque Nacional do Catimbau. A. Chapada São José. B. Cânion. C. Sítio Serra Branca. ( $\square=C$. aenea; $\square=C$. caxiensis; $\square=C$. flavifrons; 目 = C. fuscata $;$ 四 = C. tarsata $;=$ A. mellifera $)$.

Figure 3. Frequency of visits of Byrsonima gardnerana pollinators at the three study sites in the Parque Nacional do Catimbau. A. Chapada São José. B. Cânion. C. Sítio Serra Branca. $(\square=C$. aenea; $\square=C$. caxiensis; $\square=C$. flavifrons; 目 $=$ C. fuscata; $\mathbb{W}=$ C. tarsata $; \mathbf{\square}=$ A. mellifera .

medianas (com as quais raspavam as demais glândulas), com movimentos rápidos, de baixo para cima e de frente para trás (figura 4-7). Com as pernas posteriores as abelhas maiores apoiavam-se nas flores próximas para se equilibrarem.
As primeiras visitas do dia eram dedicadas à coleta do óleo floral, que se mostrava abundante. Centris aenea (figura 4) foi um dos principais visitantes de $B$. gardnerana na Chapada São José, onde também foram registradas a presença de $C$. fuscata (figura 5), $C$. 
caxiensis (figura 6), C. tarsata e $C$. flavifrons (figura 7), coletando óleo floral e pólen e Trigona spinipes, único pilhador de pólen (tabela 2). Centris flavifrons e $C$. fuscata ainda foram vistas raspando os elaióforos não só de flores, mas também de botões de B. gardnerana.

Com a diminuição da quantidade de óleo, no decorrer do tempo, as abelhas raspavam ligeiramente as glândulas e intensificavam a coleta de pólen, agarrando e vibrando as anteras. Uma nuvem de pólen era observada, sendo os grãos depositados na região ventral do tórax dessas abelhas, havendo, contudo, uma perda considerável do recurso durante a coleta realizada por Centris caxiensis, C. tarsata e Augochloropsis sp.
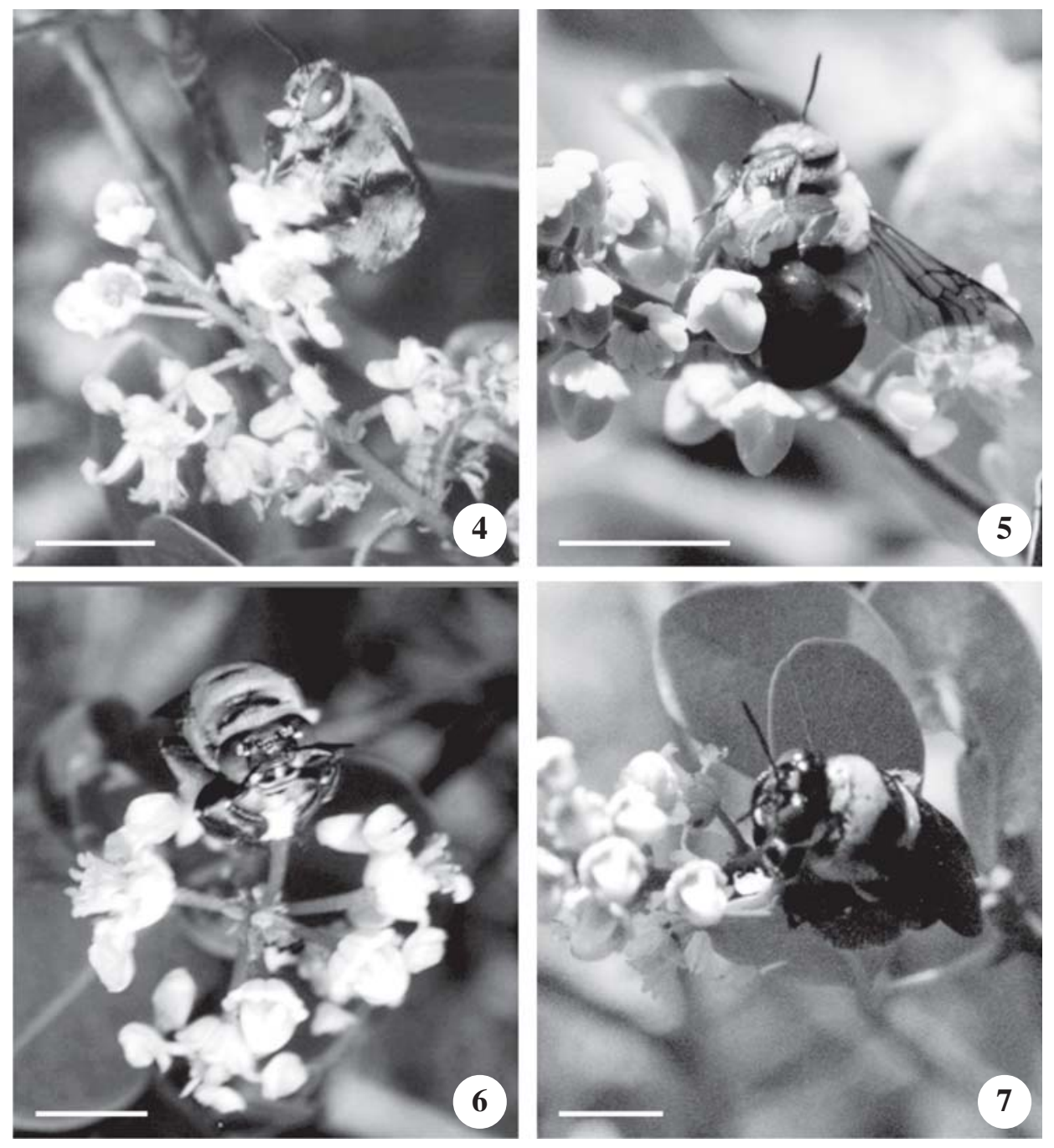

Figuras 4-7. Polinizadores de Byrsonima gardnerana. 4. Centris aenea durante coleta de óleo. 5. C. fuscata raspando elaióforos. 6. C. caxiensis. e 7. C. flavifrons, ambas envolvendo toda a flor. Escala $=1,0 \mathrm{~cm}$.

Figures 4-7. Pollinators of Byrsonima gardnerana. 4. Centris aenea while collecting oil. 5. C. fuscata scratching the elaiophores. 6. C. caxiensis and 7. C. flavifrons, both embracing the entire flower. Scale bar $=1.0 \mathrm{~cm}$. 
se enquadra no padrão cornucópia descrito por Gentry (1974), apresentando floração anual de acordo com proposições de Newstrom et al. (1994), que se caracteriza pela intensa produção de flores durante várias semanas, podendo durar de 1-5 meses.

Este padrão foi também encontrado em várias espécies do gênero para diferentes ecossistemas: Byrsonima sericea DC. em Floresta Atlântica (Teixeira \& Machado 2000); B. coccolobifolia H.B. \& K., B. crassa Nied., B. guilleminiana A. Juss., B. laxiflora Griseb., $B$. subterranea Brade \& Markgr., B. umbellata Mart. ex A. Juss., B. verbascifolia Rich. ex Juss. por Barros (1992) numa área de Cerrado e Byrsonima coccolobifolia em savana amazônica (Benezar \& Pessoni 2006), sendo possivelmente uma estratégia comum nas plantas do gênero e independente do tipo vegetacional, de solo ou clima.

A variação espaço-temporal do pico de floração observada entre as populações de $B$. gardnerana das três sub-áreas estudadas no Parna Catimbau foi também observada por Waser (1983) em diferentes populações de Ipomopsis aggregata (Polemoniaceae), podendo ser um atenuante da competição intra-específica, promovendo ainda uma maior disponibilidade do recurso floral aos seus visitantes.

A floração de B. gardnerana na Caatinga ocorreu logo após o período de chuvas na região, apresentando flores durante toda a estação seca. O mesmo foi registrado para outras espécies do gênero em diferentes ecossistemas (e.g. Albuquerque \& Rêgo 1989, Barros 1992, Teixeira \& Machado 2000, Benezar \& Pessoni 2006, Costa et al. 2006).

A produção massiva de flores após as chuvas indica que a água pode ser o fator para o desencadeamento da floração de B. gardnerana, fato constatado para outras espécies de Caatinga por Machado et al. (1997). Esta formação vegetacional apresenta forte sazonalidade e irregularidade na distribuição das chuvas, fatos que podem promover ajustes no comportamento fenológico das espécies (Schaik et al. 1993). Martins et al. (1999) comentam sobre observações de eventos de floração mais intensos em Malpighia emarginata DC. (acerola) em períodos que foram antecedidos por chuvas ou irrigação em pomares cultivados na Paraíba (nordeste do Brasil).

A floração intensa no período de estiagem também promove o deslocamento do pico de frutificação para o início da estação chuvosa, quando as sementes dispersas poderiam ter seus requerimentos hídricos necessários disponíveis, facilitando assim a germinação e o recrutamento de plântulas, como observado em diferentes espécies nos trabalhos de Janzen (1967), Frankie et al. (1974) e Primack (1987).
Morfologia floral - Os atributos florais apresentados por B. gardnerana como corola branca e zigomorfa, flores do tipo disco, antese diurna e, sobretudo, a oferta de óleo como recurso floral, permitem enquadrar a espécie na síndrome de melitofilia (sensu Faegri \& Pijl 1979). A constância destes caracteres em Malpighiaceae gera um padrão para a maioria das espécies da família, podendo ter sido resultante dos processos de co-evolução difusa com os Centridini (Anderson 1979, Buchmann 1987, Vogel 1990, Machado 2002, 2004, Alves dos Santos et al. 2007),

Sequiência da antese - A antese diurna é uma característica comumente encontrada em diferentes espécies de Malpighiaceae (e.g. Barros 1992, Freitas et al. 1999, Teixeira \& Machado 2000, Gaglianone 2000, Benezar \& Pessoni 2006, Costa et al. 2006). Os dois últimos estudos relatam abertura assincrônica de alguns botões, como observado em Byrsonima gardnerana.

A duração de sua antese foi mais prolongada que nas espécies estudadas por Barros (1992), Freitas et al. (1999) e Teixeira \& Machado (2000), nas quais as flores permaneceram abertas por, no máximo, um dia e meio. Se comparado o número de flores/inflorescência, verifica-se que $B$. gardnerana apresenta $c a .1 / 3$ do número médio de flores em relação ao apresentado pelas demais espécies de Malpighiaceae analisadas nos referidos trabalhos. Tal déficit poderia estar sendo compensado pela manutenção das flores por dois ou por até três dias (caso não sejam polinizadas), funcionando assim como um atrativo visual para as abelhas às flores, como observado em espécies de Solanaceae por Buchmann et al. (1977) e Bezerra \& Machado (2003).

Os experimentos de duração da antese realizados em B. gardnerana puderam comprovar que as alterações florais observadas ao longo da antese enquadram a espécie no segundo padrão apresentado por Gori (1983), no qual a polinização é o fenômeno responsável pela indução de mudanças no estado da flor, podendo antecipar ou adiar a senescência (Primack 1985, van Doorn 1997). A maior duração apresentada pelas flores polinizadas manualmente, em relação àquelas polinizadas naturalmente, pode ser em decorrência do fato de ter sido excluída a ação das abelhas, visto que a atividade de coleta para obtenção dos recursos florais promove atrito e oxidação decorrente da raspagem dos elaióforos, mordedura na base da pétala estandarte e vibração dos estames para a coleta de pólen.

Segundo Anderson (1979), alterações de cor da corola observadas ao longo da antese floral, apresentada por flores de Malpighiaceae, são indícios de adaptações que visam redirecionar os visitantes às flores novas, ampliando 
o número de flores visitadas (Gori 1983, van Doorn 1997), evitando ainda a remoção dos grãos já depositados no estigma e possíveis danos às estruturas reprodutivas (McDade \& Kinsman 1980).

Sistema reprodutivo - Os resultados do tratamento de polinização manual de $B$. gardnerana, associados à elevada razão pólen/óvulo $(\mathrm{P} / \mathrm{O})$ e também à alta viabilidade polínica, são indicativos de xenogamia obrigatória, como descrito por Cruden (1977), permitindo classificar a espécie como auto-incompatível.

Auto-incompatibilidade também foi constatada em outras espécies do gênero (Rêgo \& Albuquerque 1989, Teixeira \& Machado 2000, Costa et al. 2006). Este sistema reprodutivo, entretanto, não é uma característica constante na família, como observado por Barros (1992), em análise a sete espécies de Byrsonima, a qual atribui ao gênero certo grau de autocompatibilidade baseada nos testes de autopolinização manual e espontânea. Benezar $\&$ Pessoni (2006) em análise a flores de B. coccolobifolia também comprovou formação de frutos nos tratamentos de geitonogamia e autopolinização espontânea.

Visitantes florais - Abelhas fêmeas da tribo Centridini foram os únicos visitantes coletores do óleo floral de $B$. gardnerana, recurso utilizado na composição da dieta das larvas destas abelhas, junto com os grãos de pólen e/ou néctar, ou para construção e impermeabilização de seus ninhos no interior do solo (e.g. Vogel 1974, 1990, Neff \& Simpson 1981, Simpson \& Neff 1981, 1983, Buchmann 1987, Vinson et al. 1997, Michener 2000, Teixeira \& Machado 2000, Machado 2002, 2004, Aguiar et al. 2003, Aguiar \& Gaglianone 2003, Alves dos Santos et al. 2007).

Apesar da diferença da vegetação circundante, não houve variação em relação a riqueza dos Centridini nas diferentes áreas estudadas do Parque Nacional do Catimbau, ocorrendo diferença apenas entre os dois ecossistemas analisados, devido a presença de Centris nitens e C. leprieuri exclusivamente na área de Restinga.

Centris flavifrons foi registrada pela primeira vez em áreas de Caatinga por Aguiar et al. (2003). Porém, devido à área de estudo referida por estes autores situarse no limite leste da Caatinga com a Floresta Atlântica, C. flavifrons foi considerada como uma espécie de distribuição limitada a áreas de transição destes dois ecossistemas. Acoleta realizada no Parna Catimbau amplia sua distribuição para áreas do semi-árido brasileiro.

A análise do comportamento dos Centridini durante as visitas às flores de $B$. gardnerana revelou que estas abelhas podem ser consideradas os seus polinizadores efetivos, uma vez que ao visitarem sempre contataram as estruturas reprodutivas das flores. A relação entre abelhas desta tribo e flores de óleo, especialmente com a família Malpighiaceae, tem sido amplamente descrita como um processo de co-evolução difusa, caracterizada pelas adaptações morfofuncionais ocorrentes em ambos os grupos (Vogel 1974, 1990, Buchmann 1987, Simpson \& Neff 1981, 1983, Machado 2002, 2004, Alves dos Santos et al. 2007).

A presença de abelhas coletoras de óleo raspando o cálice de botões florais de B. gardnerana aponta que as glândulas são funcionais já nesta fase. A análise da anatomia e da ultra-estrutura dos elaióforos realizada por Attala \& Machado (2003) em flores de Banisteriopsis variabilis comprovou que mesmo na fase inicial de botão, as glândulas já secretam compostos lipídicos, porém em menor quantidade.

Em B. gardnerana, todas as espécies de Centris e também Augochloropsis sp. vibraram o corpo sobre as flores para coletar pólen. Este tipo de coleta através da vibração do corpo sobre as anteras em flores de deiscência não-poricida foi descrito por Buchmann (1983), o qual em seguida refere-se a Rubus parviflorus Nutt., Argemone arizonica G.B. Ownbey e Cucurbita foetidissima Kunth como espécies de flores não poricidas polinizadas por vibração (Buchmann 1985). Lopes \& Machado (1996) observaram o mesmo comportamento em Eulaema spp., Euglossa sp., Melipona scutellaris e Xylocopa suspecta em visitas às flores de Swartzia pickelli, cuja deiscência das anteras é longitudinal, fato referido também por Santos \& Machado (1998) para Augochloropsis sp. ao visitar flores de Vismia guianensis.

Em flores de óleo, Vogel \& Machado (1991) descrevem coleta de pólen por vibração por Centris hyptidis em anteras não-poricidas de Angelonia pubescens Benth. (Plantaginaceae). Em espécies de Malpighiaceae, vários representantes de Centris apresentaram este comportamento durante coleta de pólen em Byrsonima crassifolia (L.) Kunth (Rêgo \& Albuquerque 1989) e B. sericea (Teixeira \& Machado 2000), além de abelhas do gênero Epicharis (Epicharis schrottkyi) ao visitarem flores de Banisteriopsis muricata (Cav.) Cuatrec. e Stigmaphyllon lalandianum A. Juss. (Sazima \& Sazima 1989). Da mesma maneira, Xylocopa frontalis, em visitas às flores de Malpighia emarginata (Freitas et al. 1999), também efetuou coleta ativa de pólen.

A perda de grãos de pólen decorrente da vibração realizada por Centris caxiensis, $C$. tarsata e Augochloropsis sp., pode estar relacionada ao tipo de deiscência das anteras, somado à largura e não ao comprimento do corpo, como mencionado em $B$. 
crassifolia por Rêgo \& Albuquerque (1989), já que estas abelhas, independentemente do seu tamanho, são capazes de envolver todo o conjunto de estames. Um corpo delgado, entretanto, apresenta menor superfície de deposição dos grãos de pólen.

O comportamento de A. mellifera em flores de $B$. gardnerana permite classificá-la como polinizador ocasional. Esta espécie não procura o recurso floral primário oferecido aos visitantes que é o óleo, coletando, por sua vez, intensamente pólen, transferindo-o imediatamente para as corbículas, antes de se dirigirem a outras flores. Além do fato de não ser um polinizador autóctone, a coleta de pólen por estas abelhas pode diminuir a eficiência dos Centridini como polinizadores, especialmente para as espécies de Centris que entram em atividade mais tardiamente.

Se considerarmos o fato de que o maior investimento de $B$. gardnerana é na produção de óleo e que a produção de pólen nesta espécie é direcionada para a polinização/ fertilização da flor, qualquer visita que promova a intensa extração de grãos pode afetar o sucesso reprodutivo da planta. A coleta de pólen de B. gardnerana pelos Centridini só é realizada no final da manhã até o final do dia, quando várias visitas para coleta de óleo foram feitas, resultando possivelmente em polinizações bem sucedidas.

Freitas \& Paxton (1998) acrescentam ainda que, nas flores hermafroditas de Anacardium occidentale L., tanto A. mellifera quanto Centris tarsata removem aproximadamente a mesma quantidade de pólen, porém a proporção de grãos depositados no estigma floral pelo polinizador nativo é bastante superior. Gross \& Mackay (1998) ressalta que as operárias coletavam pólen previamente depositado na superfície estigmática de Melastoma affine D. Don e que o número de sementes formadas por fruto era significativamente inferior quando a última visita era realizada por A. mellifera.

A alta freqüência de A. mellifera pode também privar as espécies de Centris da quantidade necessária de pólen para a alimentação de suas larvas, uma vez que $B$. gardnerana é a principal fonte do recurso na área para essas abelhas durante o período em que a espécie floresce (observação pessoal), trazendo prejuízo a, pelo menos, cinco espécies de abelhas nativas. Em área de Caatinga, Nadia et al. (2007) também chamam atenção para a diminuição da freqüência de visitas dos polinizadores autóctones em flores de Ziziphus joazeiro Mart., mostrando que as visitas de Apis afetam negativamente a disponibilidade de néctar para os demais polinizadores.

O recrutamento em massa das abelhas melíferas, somado ao fato de A. mellifera ser uma abelha polilética, são os principais fatores causadores de impacto nas comunidades de abelhas nativas solitárias, especialmente sobre as espécies oligoléticas (Roubik 1980). O modo de recrutamento das abelhas melíferas permite que vários indivíduos de uma mesma colônia se reunam em grupos e consigam captar diferentes recursos ao mesmo tempo, além do que, espécies introduzidas são beneficiadas pela ausência de inimigos naturais (Steffan-Dewenter \& Tscharntke 2000), o que poderia explicar a intensa proliferação desta espécie no Parna Catimbau, em decorrência da implantação maciça de apiários nos últimos cinco anos.

Vários são os estudos que apontam que a introdução de A. mellifera causa potencial efeito negativo nas populações de plantas e polinizadores nativos (e.g. Gross \& Mackay 1998, Roubik 1980, 2000), diminuindo a riqueza das espécies de polinizadores nativos (e.g. Roubik 1980, 1989, Roubik \& Wolda 2001, Wilms et al. 1996), sendo ineficiente em síndromes especializadas, como é o caso das flores unissexuais, de óleo e das flores com anteras poricidas (Freitas \& Paxton 1998).

De acordo com os resultados obtidos, as flores de B. gardnerana são uma importante fonte de alimento para as abelhas especializadas nativas, cuja necessidade dos óleos florais na composição da dieta de suas larvas garante constantes visitas, assegurando a reprodução de B. gardnerana nos ecossistemas estudados.

Visto que a área de Caatinga onde o presente estudo foi realizado está inserida em uma unidade de conservação ambiental, faz-se necessário incluir no plano de manejo do Parque Nacional do Catimbau o controle do número e do tamanho dos apiários comerciais ocorrentes dentro do Parque, e nas áreas de entorno, bem como o acompanhamento das interações planta-polinizador através de estudos seqüenciais e sistematizados.

Agradecimentos - Valéria Carvalho, Tarcila Nadia e Mary Santos pela companhia e auxílio no trabalho de campo; Rita Pereira (IPA), pela identificação do material botânico; Fernando Zanella (UFPB) e Isabel Alves dos Santos (USP), pela identificação das espécies de abelhas; ao Ibama, pela licença concedida para pesquisa e a Capes (Bolsa de Mestrado concedida à E. Bezerra) e ao CNPq (Bolsas de produtividade em Pesquisa à A.V. Lopes e I.C. Machado) pelo auxílio financeiro.

\section{Referências bibliográficas}

AGUIAR, C.M.L. \& GAGLIANONE, M.C. 2003. Nesting biology of Centris (Centris) aenea Lepeletier (Hymenoptera, Apidae, Centridini). Revista Brasileira de Zoologia 20:601-606. 
AGUIAR, C.M.L. \& MARTINS, C.F. 1997. Abundância relativa, diversidade e fenologia de abelhas (Hymenoptera, Apoidea) na Caatinga, São João do Cariri, Paraíba, Brasil. Iheringia 83:151-163.

AGUIAR, C.M.L., MARTinS, C.F. \& MOURA, A.C.A. 1995. Recursos florais utilizados por abelhas (Hymenoptera, Apoidea) em área de caatinga (São João do Cariri, Paraíba). Revista Nordestina de Biologia 10:101-117.

AGUIAR, C.M.L., ZANELLA, F.C.V., MARTINS, C.F. \& CARVALHO, C.A.L. 2003. Plantas visitadas por Centris spp. (Hymenoptera: Apidae) na Caatinga para obtenção de recursos florais. Neotropical Entomology 32:247-259.

ALBUQUERQUE, P.M.C. \& RÊEGO, M.M.C. 1989. Fenologia das abelhas visitantes de murici (Byrsonima crassifolia, Malpighiaceae). Boletim do Museu Paraense Emílio Goeldi, Série Zoológica 5:163-178.

ALVES DOS SANTOS, I., MACHADO, I.C. \& GAGLIANONE, M.C. 2007. História natural das abelhas coletoras de óleo. Oecologia Brasiliensis 11: 242-255.

ANDERSON, W.R. 1979. Floral conservatism in Neotropical Malpighiaceae. Biotropica 11:219-223.

ATTALA, N.C. \& MACHADO, S.R. 2003. Anatomy and ultrastructure os Banisteriopsis variabilis Gates (Malpighiaceae) calyx glands. Acta Microscopica 12:635.

AYRES, M., AYRES JR, M., AYRES, D.L. \& SANTOS, A.S. 2003. BioEstat 3.0: aplicações estatísticas nas áreas das ciências biológicas e médicas. Sociedade Civil Mamirauá/MCT - CNPq/Conservation International, Belém.

BARROS, M.A.G. 1992. Fenologia da floração, estratégias reprodutivas e polinização de espécies simpátricas do gênero Byrsonima Rich (Malpighiaceae). Revista Brasileira de Biologia 52:343-353.

BENEZAR, R.M.C. \& PESSONI, L.A. 2006. Biologia floral e sistema reprodutivo de Byrsonima coccolobifolia (Kunth) em uma savana amazônica. Acta Amazonica 36:159-168.

BEZERRA, E.L.S. \& MACHADO, I.C. 2003. Biologia floral e sistema de polinização de Solanum stramonifolium Jacq. (Solanaceae) em remanescente de Mata Atlântica, Pernambuco. Acta Botanica Brasilica 17:249-259.

BUCHMANN, S.L. 1983. Buzz pollination in Angiosperms. In Handbook of experimental pollination biology (C.E. Jones \& R.J. Little, eds.). Scientific and Academic Editions, New York, p.73-113.

BUCHMANN, S.L. 1985. Bees use vibration to aid collection from non-poricidal flowers. Journal of the Kansas Entomologial Society 58:517-525.

BUCHMANN, S.L. 1987. The ecology of oil flowers and their bees. Annual Review of Ecology and Systematics 18:343-369.
BUCHMANN, S.L., JONES, C.E. \& COLIN, L.J. 1977. Vibratile pollination of Solanum douglasii and Solanum xantii (Solanaceae) in Southern California. The Wasman Journal Biology 35:1-25.

COSTA, C.B.N., COSTA, J.A.S. \& RAMALHO, M. 2006. Biologia reprodutiva de espécies simpátricas de Malpighiaceae em dunas costeiras da Bahia, Brasil. Revista Brasileira de Botânica 29:103-114.

CRUDEN, R.W. 1977. Pollen-ovule ratios: a conservative indicator of breeding systems in flowering plants. Evolution 31:32-46.

FAEGRI, K \& L. PIJL, L. VAN DER. 1979. The principles of pollination ecology. $3^{\text {rd }}$ ed. Pergamon Press, Oxford.

FOURNIER, L.A. 1974. Un método cuantitativo para la medición de características fenológicas en árboles. Turrialba 24:422-423.

FRANKIE, G.W., HABER, W.A. \& OPLER, B.A. 1974. Comparative phenological studies of trees in tropical wet and dry forests in the lowlands of Costa Rica. Journal of Ecology 62:881-919.

FRANKIE, G.W., HABER, W.A., OPLER, P.A. \& BAWA, K.S. 1983. Characteristics and organization of large bee pollination systems in the Costa Rican dry forest. In Handbook of experimental pollination biology (C.E. Jones \& R.J. Little, eds.). Scientific and Academic Editions, New York, p.411-447.

FREITAS, B.M. \& PAXTON, R.J. 1998. A comparison of two pollinators: Apis mellifera and an indigenous bee Centris tarsata on cashew Anacardium accidentale in its native range of NE Brazil. Journal of Applied Ecology 35:109-121.

FREITAS, B.M., ALVES, J.E., BRANDÃO, G.F. \& ARAÚJO, Z.B. 1999. Pollination requirements of West Indian cherry (Malpighia emarginata) and its putative pollinators, Centris bees, in NE Brazil. Journal of Agricultural Science 133:303-311.

GAGLIANONE, M.C. 2000. Interações de Epicharis (Apidae, Centridini) e flores de Malpighiaceae em um ecossistema de Cerrado. In: Anais do IV Encontro sobre Abelhas. Ribeirão Preto-SP, p.246-252.

GENTRY, A.H. 1974. Flowering phenology and diversity in tropical Bignoniaceae. Biotropica 6:64-68.

GORI, D.F. 1983. Post-pollination phenomena and adaptative floral changes. In Handbook of experimental pollination biology (C.E. Jones \& R.J. Little, eds.). Scientific and Academic Editions, New York, p.31-49.

GOTTSBERGER, G. 1986. Some pollination strategies in Neotropical Savannas and Forest. Plant Systematics and Evolution 152:29-45.

GROSS, C.L. \& MACKAY, D. 1998. Honeybees reduce fitness in the pioneer shrub Melastoma affine (Melastomataceae). Biological Conservation 86:169-178.

JANZEN, D.H. 1967. Synchronization of sexual reproduction of trees within the dry season in Central America. Evolution 21:620-637. 
LLOYD, D.G. 1972. Breeding systems in Cotula L. (Compositae, Anthemidae). New Physiologist 71:1181-1194.

LOPES, A.V.F., \& MACHADO, I.C. 1996. Biologia floral de Swartzia pickelii Killip ex Ducke (LeguminosaePapilionoideae) e sua polinização por Eulaema spp. (Apidae-Euglossini). Revista Brasileira de Botânica 19:17-24.

MACHADO, I.C. 2002. Flores de óleo e abelhas coletoras de óleo floral: conceitos, distribuição e revisão. In Anais do V Encontro Sobre Abelhas. Ribeirão Preto, São Paulo, p.129-135.

MACHADO, I.C. 2004. Oil-collecting bees and related plants: a review of the studies in the last twenty years and case histories of plants occurring in NE Brazil. In Solitary bees. Conservation, rearing and management for pollination (B.M. Freitas \& J.O.P. Pereira, eds.). Imprensa Universitária, Fortaleza, p.255-280.

MACHADO, I.C.S., BARROS, L.M. \& SAMPAIO, E.V.S.B. 1997. Phenology of caatinga species at Serra Talhada, PE, Northeastern Brazil. Biotropica 29:57-68.

MACHADO, I.C., VOGEL, S. \& LOPES, A.V. 2002. Pollination of Angelonia cornigera Hook. (Scrophulariaceae) by long-legged oil-collecting bees in NE Brazil. Plant Biology 4:352-359.

MARTINS, C.F. 1994. Comunidade de abelhas (Hym., Apoidea) da Caatinga e do Cerrado com elementos de Campo Rupestre do Estado da Bahia, Brasil. Revista Nordestina de Biologia 9:225-257.

MARTINS, C.F. 1995. Flora apícola e nichos tróficos de abelhas (Hym., Apoidea) na Chapada Diamantina (Lençois-BA-Brasil). Revista Nordestina de Biologia 10:119-140.

MARTINS, C.G.M., LORENZON, M.C.A. \& BAPTISTA, J.L. 1999. Eficiência de tipos de polinização em acerola. Caatinga 12:55-59.

MCDADE, L. \& KINSMAN, S. 1980. The impact of floral parasitism in two neotropical hummingbird pollinated plant species. Evolution 34:944-958.

MICHENER, C.D. 1962. As interesting method of pollen collecting by bees from flowers with tubular anthers. Revista de Biologia Tropical 10:167-175.

MICHENER, C.D. 2000. The bees of the world. The John Hopkins University Press, Baltimore, London.

MOURA, R.A., PURCHIO, A., WADA, C.S. \& ALMEIDA, T.V. 1987. Técnicas de laboratório. 3a ed. Livraria Atheneu, São Paulo.

NADIA, T.C.L., MACHADO, I.C. \& LOPES, A.V. 2007. Fenologia reprodutiva e sistema de polinização de Ziziphus joazeiro Mart. (Rhamnaceae): atuação de Apis mellifera e de visitantes florais autóctones como polinizadores. Acta Botanica Brasilica 21:835-845.

NEFF, J.L. \& SIMPSON, B.B. 1981. Oil-collecting structures in the Anthophoridae (Hymenoptera): Morphology, function and use in systematics. Journal of the Kansas Entomological Society 54:95-123.
NEWSTROM, L.E., FRANKIE, G.W. \& BAKER, H.G. 1994. A new classification for plant phenology based on flowering patterns in lowland tropical rain forest trees at La Selva, Costa Rica. Biotropica 26:141-159.

PRIMACK, R.B. 1985. Longevity of individual flowers. Annual Review of Ecology and Systematics 16:15-35.

PRIMACK, R.B. 1987. Relationships among flowers, fruits and seeds. Annual Review of Ecology and Systematics 18:409-430.

RADFORD, A.E., DICKINSON, W.C., MASSEY, J.R. \& BELL, C.R. 1974. Vascular plant systematics. Harper and Row Publishers, New York.

RAMALHO, M. \& SILVA, M. 2002. Relação Centridiniflora oleífera: influência sobre a diversidade em uma comunidade tropical. In Anais do V Encontro Sobre Abelhas. Ribeirão Preto-São Paulo, p.122-128.

RÊGO, M.M.C. \& ALBUQUERQUE, P.M.C. 1989. Comportamento das abelhas visitantes de murici, Byrsonima crassifolia (L.) Kunth, Malpighiaceae. Boletim do Museu Paraense Emílio Goeldi, Série Zoológica 5:179-193.

ROBINSOHN, I. 1924. Die farbungsreaktion der narbe, stigmatochromie, als morpho-biologische blütenuntersuchungs methode. Sitzungsberichten Akademischer Wissenschaft Wien, Mathematics, Abteilung I 133:180-213.

RODAL, M.J.N., ANDRADE, K.V.A., SALES, M.F. \& GOMES, A.P.S. 1998. Fitossociologia do componente lenhoso de um refúgio vegetacional no município de Buíque, Pernambuco. Revista Brasileira de Biologia 58:517-526.

ROUBIK, D.W. 1980. Foraging behaviour of competing africanized honey bees and stingless bees. Ecology 61:836-845.

ROUBIK, D.W. 1989. Ecology and natural history of tropical bees. Cambridge University Press, New York.

ROUBIK, D.W. 2000. Pollination system stability in Tropical America. Conservation Biology 14:1235-1236.

ROUBIK, D.W. \& WOLDA, H. 2001. Do competing honey bees matter? Dynamics and abundance of native bees before and after honey bee invasion. Population Ecology 43:53-62.

SANTOS, M.J.L. \& MACHADO, I.C. 1998. Biologia floral e heterostilia em Vismia guianensis (Aubl.) Choisy (Clusiaceae). Acta Botanica Brasilica 12:451-464.

SAZIMA, M. \& SAZIMA, I. 1989. Oil-gathering bees visit flowers of eglandular morphs of the oil-producing Malpighiaceae. Botanica Acta 102:106-111.

SCHAIK, C.P., TERBORGH, J.W. \& WRIGHT, S.J. 1993. The phenology of tropical forest: adaptative significance and consequences for primary consumers. Annual Annual Review of Ecology and Systematics 24:353-377.

SIGRIST, M.R. \& SAZIMA, M. 2004. Pollination and reproductive biology of twelve species of neotropical Malpighiaceae: Stigma morphology and its implications for the breeding systems. Annals of Botany 94:33-41. 
SILVA, M.C.M. \& MARTINS, C.F. 1999. Flora apícola e relações tróficas de abelhas (Hymenoptera: Apoidea) em uma área de restinga (Praia de Intermares, Cabedelo - PB, Brasil). Principia 7:40-51.

SIMPSON, B.B. \& NEFF, J.L. 1981. Floral rewards: alternatives to pollen and nectar. Annals of the Missouri Botanical Garden 68:301-322.

SIMPSON, B.B. \& NEFF, J.L. 1983. Evolution and diversity of floral rewards. In Handbook of experimental pollination biology (C.E. Jones \& R.J. Little, eds.). Scientific and Academic Editions, New York, p.142-159.

SNE. 2002. Projeto técnico para criação do Parque Nacional do Catimbau/PE. Versão final. Sociedade Nordestina de Ecologia (SNE), Recife.

STEFFAN-DEWENTER, I. \& TSCHARNTKE, T. 2000. Resource overlap and possible competition between honey bees and wild bees in central Europe. Oecologia 122:288-296.

SUDENE. 1990. Dados pluviométricos mensais do Nordeste. Sudene, Recife.

TEIXEIRA, L.A.G. \& MACHADO, I.C. 2000. Sistemas de polinização e reprodução de Byrsonima sericea DC (Malpighiaceae). Acta Botanica Brasilica 14:347-357.

VAN DOORN, W.G. 1997. Effects of pollination on floral attraction and longevity. Journal of Experimental Botany 48:1615-1622.

VINSON, S.B., WILLIAMS, H.J., FRANKIE, G.W. \& SHRUM, G. 1997. Floral lipid chemistry of Byrsonima crassifolia (Malpighiaceae) and a use of floral lipids by Centris bees (Hymenoptera: Apidae). Biotropica 29: 76-83.
VOGEL, S. 1974. Ölblumen und ölsammelnde Bienen. Tropische und Subtropische Pflanzenwelt 7:285-547.

VOGEL, S. 1990. History of the Malpighiaceae in the light of the pollination ecology. Memoirs of the New York Botanical Garden 55:130-142.

VOGEL, S. \& MACHADO, I.C. 1991. Pollination of four sympatric species of Angelonia (Scrophulariaceae) by oil-collecting bees in NE Brazil. Plant Systematics and Evolution 178:153-178.

WASER, N.M. 1983. Competition for pollination and floral character differences among sympatric plant species: a review of evidence. In Handbook of experimental pollination biology (C.E. Jones \& R.J. Little, eds.). New York, Scientific and Academic Editions, p.277-293.

WILLE, A. 1963. Behavioural adaptations of bees for pollen collecting from Cassia flowers. Revista de Biologia Tropical 11:205-210.

WILMS, W., IMPERATRIZ-FONSECA, V.L. \& ENGELS, W. 1996. Resource partitioning between highly eusocial bees and possible impact of the introduced Africanized honey bee on native stingless bees in the Brazilian Atlantic forest. Stud. Neotropical Fauna \& Environment 31:137-151.

ZANELLA, F.C.V. 2000a. Padrões de distribuição geográfica das espécies de abelhas que ocorrem na Caatinga (NE do Brasil). In Anais do IV Encontro Sobre Abelhas. Ribeirão Preto-São Paulo, p.197-203.

ZANELLA, F.C.V. 2000b. The bees of the Caatinga (Hymenoptera, Apoidea, Apiformes): a species list and comparative notes regarding their distribution. Apidologie 31:579-592. 